\title{
Oncologic outcomes in robot-assisted radical cystectomy: Where do we stand in 2021?
}

\author{
Brady L. Miller, Mark Pachorek, Andre-Philippe Sam, Bertram Yuh, Clayton S. Lau \\ Division of Urologic Oncology, Department of Surgery, City of Hope National Comprehensive Cancer Center, Duarte, CA 91010, \\ USA.
}

Correspondence to: Dr. Clayton S. Lau, Division of Urology, Department of Surgery, City of Hope, 1500 E. Duarte Rd, MALP \#1211, Duarte, CA 91010, USA. E-mail: cllau@coh.org

How to cite this article: Miller BL, Pachorek M, Sam AP, Yuh B, Lau CS. Oncologic outcomes in robot-assisted radical cystectomy: Where do we stand in 2021? Mini-invasive Surg 2021;5:24. https://dx.doi.org/10.20517/2574-1225.2021.25

Received: 24 Feb 2021 First Decision: 31 Mar 2021 Revised: 7 Apr 2021 Accepted: 12 Apr 2021 Published: 8 May 2021

Academic Editor: Giulio Belli Copy Editor: Xi-Jun Chen Production Editor: Xi-Jun Chen

\begin{abstract}
Robot-assisted radical cystectomy is an alternative to the standard open surgical approach and has been increasingly used to surgically treat bladder cancer. Data on oncologic outcomes for the robotic approach have matured, and now intermediate and long-term oncologic outcomes are available. This review focuses on oncologic outcomes of the robotic approach with a focus on recent data and high-quality studies. Based on the current literature available, there are no consistent differences between the robotic and open approaches with respect to positive margin rates, lymph node yields, recurrence patterns, or recurrence free, cancer-specific, and overall survival. If oncologic surgical principles are adhered to, excellent oncologic outcomes are achievable with the robotic approach.
\end{abstract}

Keywords: Urinary bladder neoplasms, radical cystectomy, robotic radical cystectomy, oncologic outcomes, robotics, recurrence, survival

\section{INTRODUCTION}

Radical cystectomy and pelvic lymph node dissection is standard of care for surgically eligible patients with non-metastatic muscle-invasive bladder cancer, and is a preferred treatment for select patients with high risk of non-muscle invasive disease ${ }^{[1,2]}$. While open radical cystectomy has been the recognized gold standard for years, robot-assisted radical cystectomy (RARC) has become increasingly popular. Initially 
described by Menon et al. ${ }^{[3]}$ in 2003, utilization of RARC increased from $0.7 \%$ in 2002 to $18.5 \%$ in 2012 in the United States ${ }^{[4]}$. Advantages of the robotic approach relative to open radical cystectomy (ORC) include reduced blood loss, favorable transfusion rate and shorter length of stay ${ }^{[5]}$.

Here, we review pertinent oncologic outcomes in the current RARC literature. We queried the PubMed electronic database in January 2021 for studies that report on oncologic outcomes for RARC. An emphasis was placed on randomized controlled trials, as well as contemporary comparative open approach cohorts, large single institution surgical series, multi-center initiatives and systematic reviews. A list of the major studies considered in this review is found in Table 1.

\section{NODAL YIELD}

Lower nodal yield and positive surgical margin status are independently associated with worse OS after adjustment for neoadjuvant chemotherapy and pathologic factors. In fact, nodal yields of 10-14 have been proposed as a marker of surgical quality ${ }^{[33]}$. Professional guidelines and best practice statements are less quantitatively prescriptive ${ }^{[1,34]}$, as patient, clinical and pathologic factors can influence lymph node yield. In a 2015 systematic review, Yuh et al. ${ }^{[35]}$ assessed 105 papers and found that median yield for a robotic approach was 19 lymph nodes (range: 3-55) with cumulative analyses finding no difference vs. ORC. Nodal yields are directly related to the surgical dissection template chosen, whether standard or extended. Among robotic surgeons, high volume surgeons and institutional volume were independently associated with performance of extended template dissections ${ }^{[36]}$.

Several RCTs have found comparable nodal yields between RARC and ORC [Table 2]. Nix et al. ${ }^{[11]}$ found mean LN yields of 19 vs. 18 in RARC vs. ORC $(P=0.51)$ using a standard dissection template. In the largest clinical trial, RAZOR investigators found similar median lymph node yields of 23.3 for RARC with $51 \%$ utilizing an extended template, and 25.7 for ORC with $55 \%$ utilizing an extended template $(P=0.13)^{[6]}$. Other smaller RCTs reported similar findings ${ }^{[8,9]}$. Several recent meta-analyses did not assess nodal yield ${ }^{[37,38]}$.

Considering the abundance of data, adequate lymph node yields are achievable via robotic platforms, including extended and super extended templates. Maintenance of oncologic principles including performance of a meticulous dissection within pre-defined anatomic boundaries of a template appears to be more important than surgical approach.

\section{POSITIVE MARGIN RATE}

Positive surgical margin (PSM) rate is a measure of local disease burden, an independent predictor of survival, and can be a measure of surgical quality ${ }^{[3,39,40]}$. Early criticism of minimally invasive approaches was that there was risk of higher positive margin rates in locally advanced tumors, as evidenced by a single non-controlled, non-comparative retrospective study ${ }^{[32]}$. It was theorized that the lack of tactile feedback and learning curve was potential explanations $s^{[35,41]}$.

These early criticisms have largely been refuted. A systematic review showed that PSM rate was low in pT2 disease $(<1.5 \%)$ and $0 \%-25 \%$ in pT3 disease or higher, without any significant difference between ORC and RARC in a cumulative analysis of 17 studies $^{[35]}$. Interestingly, PSM did not appear to decrease with sequential case numbers or institutional volume ${ }^{[32]}$, a finding that may reflect surgeons' willingness to take on more difficult cases with experience ${ }^{[35]}$. As a result of these early robotic data and historical open cystectomy series, acceptable PSM rates for robotic surgeons were proposed as $<3 \%$ for $\mathrm{pT} 2,<10 \%$ for $\mathrm{pT} 3$, $<25 \%$ for $\mathrm{pT} 4$ and $<7 \%$ overall $^{[3,40]}$. 
Table 1. Selected studies evaluating oncologic outcomes after robot-assisted radical cystectomy

\begin{tabular}{|c|c|c|c|c|c|c|}
\hline Ref. & Year & Comparison & $\begin{array}{l}\text { Study } \\
\text { design }\end{array}$ & Setting & Primary outcome & Pertinent secondary outcome(s) \\
\hline \multicolumn{7}{|l|}{ Comparative studies, randomized } \\
\hline RAZOR trial, multiple authors ${ }^{[6,7]}$ & $\begin{array}{l}2020 \\
2018\end{array}$ & ORC vs. RARC & $\mathrm{RCT}$ & Multi center & 2-year PFS & TTR, PFS, OS \\
\hline CORAL trial, Khan et al. ${ }^{[8]}$ & 2020 & $\begin{array}{l}\text { ORC vs. RARC vs. } \\
\text { LRC }\end{array}$ & $\mathrm{RCT}$ & Single center & 5-year RFS, CSS, OS & Surgical margin, recurrence patterns \\
\hline Bochner et al. ${ }^{[9]}$ & 2018 & ORC vs. RARC & $\mathrm{RCT}$ & Single center & 90-day complication & RFS, CSS, OS, recurrence patterns \\
\hline Parekh et al. ${ }^{[10]}$ & 2012 & ORC vs. RARC & $\mathrm{RCT}$ & Single center & $\begin{array}{l}\text { Surgical margin } \\
\text { Total lymph node yield }\end{array}$ & $\begin{array}{l}\text { Quality of life } \\
\text { Functional recovery }\end{array}$ \\
\hline Nix et al. ${ }^{[11]}$ & 2010 & ORC vs. RARC & $\mathrm{RCT}$ & Single center & Lymph node yield & $\begin{array}{l}\text { Demographics, perioperative, pathologic results, narcotic } \\
\text { use }\end{array}$ \\
\hline \multicolumn{7}{|c|}{ Comparative studies, non-randomized } \\
\hline RACE study, Wijburg et al. ${ }^{[12]}$ & 2021 & ORC vs. RARC & Prospective & Multi center & 90-day complication & $\begin{array}{l}\text { HRQOL, complications, clinical outcomes including } \\
\text { surgical margin }\end{array}$ \\
\hline Asil et al. ${ }^{[13]}$ & 2021 & ORC vs. RARC & Retrospective & Multi center & Intraoperative and postoperative endpoints & Surgical margin, lymph node yield \\
\hline Ip et al. ${ }^{[14]}$ & 2020 & ORC vs. RARC & Retrospective & Single center & RFS, OS & Perioperative and pathologic outcomes \\
\hline Zhang et al. ${ }^{[15]}$ & 2020 & ORC vs. RARC & Retrospective & Single center & Perioperative outcomes, complications & Pathologic outcomes, overall survival \\
\hline Faraj et al. ${ }^{[16]}$ & 2019 & ORC vs. RARC & Retrospective & Single center & RFS, OS & Recurrence patterns, predictors of primary outcome \\
\hline Moschini et al. ${ }^{[17]}$ & 2019 & ORC vs. RARC & Retrospective & Multicenter & Surgical margin status & Predictors of surgical margin status \\
\hline Simone et al. ${ }^{[18]}$ & 2018 & $\begin{array}{l}\text { ORC vs. RARC, } \\
\text { ICUD only }\end{array}$ & Retrospective & Single center & RFS, CSS, OS & Complications, perioperative and pathologic outcomes \\
\hline Hanna et al. ${ }^{[19]}$ & 2018 & ORC vs. RARC & Retrospective & $\begin{array}{l}\text { Population } \\
\text { registry }\end{array}$ & Intraoperative and postoperative endpoints & Descriptors and predictors of robotic surgical approach \\
\hline Gandagli et al. ${ }^{[20]}$ & 2016 & ORC vs. RARC & Retrospective & Multi center & RFS, CSS, OS & $\begin{array}{l}\text { Complications, perioperative and pathologic outcomes, } \\
\text { recurrence }\end{array}$ \\
\hline Tan et al. ${ }^{[21]}$ & 2016 & ORC vs. RARC & Retrospective & Single center & RFS & Recurrence patterns, CSS, OS \\
\hline Matulewicz et al..$^{[22]}$ & 2016 & ORC vs. RARC & Retrospective & $\begin{array}{l}\text { Population } \\
\text { registry }\end{array}$ & Surgical margin status, lymph node yield & Primary outcome variables as predictors of survival \\
\hline Nguyen et al. ${ }^{[23]}$ & 2015 & ORC vs. RARC & Retrospective & Single center & RFS & Recurrence patterns at 2 years \\
\hline Atmaca et al. ${ }^{[24]}$ & 2015 & $\begin{array}{l}\text { ORC vs. RARC, } \\
\text { ICUD only }\end{array}$ & Retrospective & Single center & $\begin{array}{l}\text { Demographics, functional, intraoperative } \\
\text { outcomes }\end{array}$ & Surgical margin, lymph node yield \\
\hline \multicolumn{7}{|l|}{ Non-comparative studies } \\
\hline IRCC, Elsayed et al. ${ }^{[25]}$ & 2021 & RARC only & Retrospective & Multicenter & RFS, LRFS, DMFS, OS & Recurrence patterns, predictors of recurrent free survival \\
\hline Brassetti et al. ${ }^{[26]}$ & 2020 & RARC, ICUD only & Retrospective & Multicenter & RFS, CSS, OS & Surgical margin, lymph node yield, predictors of survival \\
\hline IRCC, Hussein et al. ${ }^{[27]}$ & 2019 & RARC only & Retrospective & Multicenter & 10-year RFS, CSS, OS & Surgical margin, lymph node yield, predictors of survival \\
\hline
\end{tabular}




\begin{tabular}{|c|c|c|c|c|c|c|}
\hline IRCC, Hussein et al. ${ }^{[28]}$ & 2017 & RARC only & Retrospective & Multicenter & $\begin{array}{l}\text { Incidence of early oncologic failure (any disease } \\
\text { relapse }<3 \mathrm{mo} \mathrm{s} / \mathrm{p} \text { RARC) }\end{array}$ & $\begin{array}{l}\text { Recurrence patterns, adherence to oncologic principles, } \\
\text { predictors of early oncologic failure }\end{array}$ \\
\hline ERUS, Collins et al. ${ }^{[29]}$ & 2017 & RARC, ICUD only & Retrospective & Multicenter & RFS & Recurrence patterns \\
\hline IRCC, Raza et al. ${ }^{[30]}$ & 2015 & RARC only & Retrospective & Multicenter & 5-year RFS, CSS, OS & Surgical margin, lymph node yield, predictors of survival \\
\hline IRCC, Hellenthal et al. ${ }^{[31]}$ & 2011 & RARC only & Retrospective & Multicenter & $\begin{array}{l}\text { Proportion of RARC w/lymphadenectomy } \\
\text { performed }\end{array}$ & $\begin{array}{l}\text { Lymph node yield, predictors of lymphadenectomy } \\
\text { performance }\end{array}$ \\
\hline IRCC, Hellenthal et al. ${ }^{[32]}$ & 2010 & RARC only & Retrospective & Multicenter & Surgical margin status & Predictors of surgical margin status \\
\hline
\end{tabular}

RAZOR: Randomized open vs. robotic cystectomy; ORC: open radical cystectomy; RARC: robotic assisted radical cystectomy; RCT: randomized controlled trial; TTR: time to recurrence; RFS: recurrence free survival; PFS: progression free survival; CSS: cancer specific survival; OS: overall survival; CORAL: controlled three-arm trial of Open, Robotic, and laparoscopic radical cystectomy; RACE: radical cystectomy evaluation; HRQOL: health-related quality of life; IRCC: International Robotic Cystectomy consortium; LRFS: local recurrence free survival; DMFS: distant metastasis free survival; ICUD: intracorporeal urinary diversion; ERUS: European Association of Urology Robotic Urology Section; LRC: laparoscopic radical cystectomy.

Since then, multiple RCTs and retrospective comparative studies offer additional insight that robotic cystectomy can meet these standards of surgical quality. The RAZOR trial showed overall PSM rates of 5\% (ORC) vs. 6\% (RARC), $P=0.6$ without any difference in pathologic stage between the groups. Of those with PSMs, 7/9 (78\%) in RARC and 5/7 (71\%) in ORC were T3 or above ${ }^{[6]}$. Two smaller RCTs also found no difference in PSM rate between open and robotic approaches ${ }^{[8,9]}$. A meta-analysis compiling 541 patients from RCTs showed no difference in PSM rates between RARC and ORC (RR $=1.2 ; 95 \%$ CI: 0.6-2.4 ${ }^{[37]}$. Additionally, one non-randomized comparative study found significantly increased PSM rate for ORC (18\%) vs. RARC (6\%) in an inversed probability weighted population despite similar pathologic staging, though when further specified by site of positive margin these results were not significantly different ${ }^{[12]}$. Multiple other non-randomized comparative studies have not found significant differences in PSM rate by approach ${ }^{[13-17,1,2,20,22-24]}$.

Collectively, the above data suggest favorable PSM rates are achievable via the robotic platform and are in alignment with standards of surgical quality set forth by best practices statements ${ }^{[34]}$. Regardless of surgical approach, the largest determinant of PSM rates is local disease stage.

\section{RECURRENCE PATTERNS}

Recurrence of bladder cancer after radical cystectomy is dependent on tumor and nodal stage, and ranges from $20 \%$ to $30 \%$ in pT2 disease, $40 \%$ for pT3, > 50\% for $\mathrm{pT} 4$ and approximately $70 \%$ in $\mathrm{pN} 1$ disease or greater ${ }^{[2]}$. Other independent predictors of tumor recurrence include lymphovascular invasion and positive soft tissue margins ${ }^{[43]}$. Recurrences generally occur within the first 2-3 years and predict worse overall survival (OS) ${ }^{[44]}$.

Recurrence is generally classified as local, often referring to the cystectomy bed and within the pelvic lymph node template, or distant. Atypical patterns in MIS generally refer to peritoneal carcinomatosis, abdominal wall/port site metastases and extra pelvic lymph node recurrences, which have been described but are rare. In fact, a systematic review of 1094 studies found only 5 that reported port site metastasis ${ }^{[45]}$. Proposed contributors of atypical recurrence patterns in MIS include depressive local immunologic factors and/or enhanced tumor dissemination related to pneumoperitoneum, breach of oncologic operative principles, 
Table 2. Oncologic outcomes from selected studies after robot-assisted radical cystectomy

\begin{tabular}{|c|c|c|c|c|c|c|c|c|}
\hline Ref. and study acronym & Year & Surgical approach & Cases, $(n)$ & PSM, $n(\%)$ & Lymph node yield, mean (SD) or median (IQR or range) & RFS & CSS & OS \\
\hline \multicolumn{9}{|l|}{ Comparative studies, randomized } \\
\hline \multirow[t]{2}{*}{ RAZOR trial, multiple authors ${ }^{[6,7]}$} & 2020,2018 & ORC & 152 & $7(5)$ & $25.7($ SD 14.5) & $65 \%, 3 \mathrm{yr}$ & $\mathrm{nr}$ & $69 \%, 3 \mathrm{yr}$ \\
\hline & & RARC & 150 & $9(6)$ & $23.3($ SD 12.5) & $68 \%, 3 \mathrm{yr}$ & $\mathrm{nr}$ & $74 \%, 3 \mathrm{yr}$ \\
\hline \multirow[t]{3}{*}{ CORAL trial, Khan et al. ${ }^{[8]}$} & 2020 & ORC & 20 & $2(10)$ & 18.5 (IQR 14-25) & $60 \%, 5 \mathrm{yr}$ & $64 \%, 5 \mathrm{yr}$ & $55 \%, 5 \mathrm{yr}$ \\
\hline & & RARC & 20 & $3(15)$ & 14.5 (IQR 11-21) & $58 \%, 5 \mathrm{yr}$ & $68 \%, 5 \mathrm{yr}$ & $65 \%, 5 \mathrm{yr}$ \\
\hline & & LRC & 19 & $1(5)$ & 15.5 (IQR 12-22) & $71 \%, 5 \mathrm{yr}$ & $69 \%, 5 \mathrm{yr}$ & $61 \%, 5 \mathrm{yr}$ \\
\hline \multirow[t]{2}{*}{ Bochner et al. $^{[9]}$} & 2018 & ORC & 58 & $3(5)$ & 29 (IQR 22-38) & $59 \%, 5 \mathrm{yr}$ & $80 \%, 5 \mathrm{yr}^{\#}$ & $65 \%, 5 \mathrm{yr}^{\#}$ \\
\hline & & RARC & 60 & $2(3)$ & 31 (IQR 23-37) & $64 \%, 5 \mathrm{yr}$ & $75 \%, 5 \mathrm{yr}^{\#}$ & $65 \%, 5 \mathrm{yr}^{\#}$ \\
\hline \multirow[t]{2}{*}{ Parekh et al. ${ }^{[10]}$} & 2012 & ORC & 20 & $1(5)$ & 23 (IQR 15-28) & $\mathrm{nr}$ & $\mathrm{nr}$ & $\mathrm{nr}$ \\
\hline & & RARC & 20 & $1(5)$ & 11 (IQR 9-22) & $\mathrm{nr}$ & $\mathrm{nr}$ & $\mathrm{nr}$ \\
\hline \multirow[t]{2}{*}{ Nix et al. ${ }^{[11]}$} & 2010 & ORC & 20 & $0(0)$ & 18 (range 8-30) & $\mathrm{nr}$ & $\mathrm{nr}$ & $\mathrm{nr}$ \\
\hline & & RARC & 21 & $0(0)$ & 19 (range 12-30) & $\mathrm{nr}$ & $\mathrm{nr}$ & $\mathrm{nr}$ \\
\hline \multicolumn{9}{|c|}{ Comparative studies, non-randomized } \\
\hline \multirow[t]{2}{*}{ RACE study, Wijburg et al. ${ }^{[12]}$} & 2021 & ORC & 168 & $\operatorname{nr}(18)^{\star}$ & 13 (IQR 9-18) & $75 \%, 1 \mathrm{yr}$ & $\mathrm{nr}$ & $\mathrm{nr}$ \\
\hline & & RARC & 180 & $\operatorname{nr}(6)$ & 15 (IQR 11-21) & $76 \%, 1 \mathrm{yr}$ & $\mathrm{nr}$ & $\mathrm{nr}$ \\
\hline \multirow[t]{2}{*}{ Asil et al. ${ }^{[13]}$} & 2021 & ORC & 31 & $1(3)$ & $22(n r)$ & $\mathrm{nr}$ & $\mathrm{nr}$ & $\mathrm{nr}$ \\
\hline & & RARC & 61 & $9(15)$ & Range 22-25 & $\mathrm{nr}$ & $\mathrm{nr}$ & $\mathrm{nr}$ \\
\hline \multirow[t]{2}{*}{ Ip et al. ${ }^{[14]}$} & 2020 & ORC & 159 & $23(14)$ & $20(\operatorname{SD~} 14)^{\star}$ & $75 \%, 5 \mathrm{yr}^{\#}$ & $\mathrm{nr}$ & $65 \%, 5 \mathrm{yr}^{\#}$ \\
\hline & & RARC & 73 & $8(11)$ & $12(\mathrm{SD} 8)$ & $80 \%, 5 \mathrm{yr}^{\#}$ & $\mathrm{nr}$ & $70 \%, 5 \mathrm{yr}^{\#}$ \\
\hline \multirow[t]{2}{*}{ Zhang et al. ${ }^{[15]}$} & 2020 & ORC & 272 & $22(8)$ & $\mathrm{nr}$ & $\mathrm{nr}$ & $\mathrm{nr}$ & $55 \%, 5 \mathrm{yr}$ \\
\hline & & RARC & 676 & $34(5)$ & $\mathrm{nr}$ & $\mathrm{nr}$ & $\mathrm{nr}$ & $58 \%, 5 \mathrm{yr}$ \\
\hline \multirow[t]{2}{*}{ Faraj et al. ${ }^{[16]}$} & 2019 & ORC & 278 & $15(5)$ & $12($ IQR 9-18)* & $63 \%, 10 \mathrm{yr}$ & $\mathrm{nr}$ & $46 \%, 10 \mathrm{yr}$ \\
\hline & & RARC & 203 & $7(3)$ & 18 (IQR 14-24) & $70 \%, 10 \mathrm{yr}$ & $\mathrm{nr}$ & $40 \%, 10 \mathrm{yr}$ \\
\hline \multirow[t]{2}{*}{ Moschini et al. ${ }^{[17]}$} & 2019 & ORC & 1666 & $160(10)$ & $16(10-24)$ & $\mathrm{nr}$ & $\mathrm{nr}$ & $\mathrm{nr}$ \\
\hline & & RARC & 870 & $112(13)$ & $18(12-25)$ & $\mathrm{nr}$ & $\mathrm{nr}$ & $\mathrm{nr}$ \\
\hline \multirow[t]{2}{*}{ Simone et al. ${ }^{[18]}$} & 2018 & RARC, ICUD only & 64 & $0(0)$ & 33.4 (SD 12.3) & $79 \%, 4 \mathrm{yr}$ & $85 \%, 4 \mathrm{yr}$ & $82 \%, 4 \mathrm{yr}$ \\
\hline & & ORC & 46 & $0(0)$ & $31.3($ SD 14.6) & $73 \%, 4 \mathrm{yr}$ & $86 \%, 4 \mathrm{yr}$ & $80 \%, 4 \mathrm{yr}$ \\
\hline \multirow[t]{2}{*}{ Hanna et al. ${ }^{[19]}$} & 2018 & ORC & 7513 & $(10.7)$ & $12($ IQR 7-20)* & $\mathrm{nr}$ & $\mathrm{nr}$ & $\mathrm{nr}$ \\
\hline & & RARC & 2048 & (9.3) & 17 (IQR 10-25) & $\mathrm{nr}$ & $\mathrm{nr}$ & $\mathrm{nr}$ \\
\hline \multirow[t]{2}{*}{ Gandagli et al. ${ }^{[20]}$} & 2016 & ORC & 230 & $31(13)$ & 13 (IQR 9-17) & $57 \%, 5 \mathrm{yr}$ & $62 \%, 5 \mathrm{yr}$ & $58 \%, 5 \mathrm{yr}$ \\
\hline & & RARC & 138 & $12(9)$ & 12 (IQR 8-17) & $54 \%, 5 \mathrm{yr}$ & $74 \%, 5 \mathrm{yr}$ & $59 \%, 5 \mathrm{yr}$ \\
\hline Tan et al. ${ }^{[21]}$ & 2016 & ORC & 90 & $17(19)^{\star}$ & $12.6(\mathrm{SD} 10.9)$ & $70 \%, 2 \mathrm{yr}$ & $81 \%, 2 \mathrm{yr}$ & $74 \%, 2 \mathrm{yr}$ \\
\hline
\end{tabular}




\begin{tabular}{|c|c|c|c|c|c|c|c|c|}
\hline & & RARC & 94 & $6(8)$ & 14.9 (SD 10.0) & $79 \%, 2 \mathrm{yr}$ & $84 \%, 2 \mathrm{yr}$ & $84 \%, 2 \mathrm{yr}$ \\
\hline \multirow{2}{*}{ Matulewicz et al. ${ }^{[22]}$} & 2016 & ORC & 9639 & (13) & $11($ IQR 5-19)* & $\mathrm{nr}$ & $\mathrm{nr}$ & $\mathrm{nr}$ \\
\hline & & RARC & 2397 & (11) & 16 (IQR 9-25) & $\mathrm{nr}$ & $\mathrm{nr}$ & $\mathrm{nr}$ \\
\hline \multirow[t]{2}{*}{ Nguyen et al. ${ }^{[23]}$} & 2015 & ORC & 120 & $15(13)^{\star}$ & 20 (IQR 11-27) & $60 \%, 5 \mathrm{yr}^{\#}$ & $\mathrm{nr}$ & $\mathrm{nr}$ \\
\hline & & RARC & 263 & $16(6)$ & 21 (IQR 13-28) & $70 \%, 5 \mathrm{yr}^{\#}$ & $\mathrm{nr}$ & $\mathrm{nr}$ \\
\hline \multirow[t]{2}{*}{ Atmaca et al. ${ }^{[24]}$} & 2015 & ORC & 42 & $1(2)$ & $17(S D 13.5)$ & $\mathrm{nr}$ & $\mathrm{nr}$ & $\mathrm{nr}$ \\
\hline & & RARC, ICUD only & 32 & $2(6)$ & 25 (SD 9.7) & $\mathrm{nr}$ & $\mathrm{nr}$ & $\mathrm{nr}$ \\
\hline \multicolumn{9}{|l|}{ Non-comparative studies } \\
\hline IRCC, Elsayed et al. ${ }^{[25]}$ & 2021 & RARC only & 2107 & $\mathrm{nr}$ & $\mathrm{nr}$ & $66 \%, 5 \mathrm{yr}$ & $\mathrm{nr}$ & $60 \%, 5 \mathrm{yr}$ \\
\hline Brassetti et al. ${ }^{[26]}$ & 2020 & RARC, ICUD only & 113 & $9(8)$ & 36 (IQR 28-45) & $58 \%, 5 \mathrm{yr}$ & $61 \%, 5 \mathrm{yr}$ & $54 \%, 5 \mathrm{yr}$ \\
\hline IRCC, Hussein et al. ${ }^{[27]}$ & 2019 & RARC only & 446 & $30(7)$ & 14 (IQR 9-22) & $59 \%, 10 \mathrm{yr}$ & $65 \%, 10 \mathrm{yr}$ & $35 \%, 10 \mathrm{yr}$ \\
\hline ERUS, Collins et al. ${ }^{[29]}$ & 2017 & RARC, ICUD only & 717 & $34(4)$ & 18 (IQR 13-25) & $75 \%, 2 \mathrm{yr}$ & & \\
\hline IRCC, Raza et al. ${ }^{[30]}$ & 2015 & RARC only & 702 & $55(8)$ & 16 (IQR 10-24) & $67 \%, 5 \mathrm{yr}$ & $75 \%, 5 \mathrm{yr}$ & $50 \%, 5 \mathrm{yr}$ \\
\hline IRCC, Hellenthal, et al. ${ }^{[31]}$ & 2011 & RARC only & 527 & $\mathrm{nr}$ & 17.8 (range 0-68) & $\mathrm{nr}$ & $\mathrm{nr}$ & $\mathrm{nr}$ \\
\hline IRCC, Hellenthal, et al. ${ }^{[32]}$ & 2010 & RARC only & 513 & $35(6.8)$ & $\mathrm{nr}$ & $\mathrm{nr}$ & $\mathrm{nr}$ & $\mathrm{nr}$ \\
\hline
\end{tabular}

\#Visual estimate based on Kaplan Meier curves provided in paper (specific numbers not provided by reference in text). "P< 0.05. PSM: Positive surgical margin; SD: standard deviation; IQR: interquartile range; RFS: recurrence free survival; CSS: cancer-specific survival; OS: overall survival; ORC: open radical cystectomy; RARC: robotic assisted radical cystectomy; LRC: laparoscopic radical cystectomy; nr: not reported; ICUD: intracorporeal urinary diversion: RAZOR: randomized open vs, robotic cystectomy; CORAL: controlled three-arm trial of open, robotic, and laparoscopic radical cystectomy; RACE: radical cystectomy evaluation; IRCC: International Robotic Cystectomy Consortium; ERUS: European Association of Urology Robotic Urology Section.

or variant lymphatic dissemination related to robotic technique ${ }^{[23]}$.

Nguyen et al. ${ }^{[23]}$ reported atypical patterns of recurrence in a non-randomized single center comparative study of ORC vs. RARC, including higher incidence of peritoneal carcinomatosis $(21 \%$ vs. $8 \%)$ and extra pelvic lymph node ( $23 \%$ vs. $15 \%)$ [Table 3]. However, the denominator of these estimated proportions was distant recurrences and not overall recurrence, as is typically reported. It was additionally notable that distant recurrences were not significantly different between the two approaches, and the authors noted that selection bias may have contributed to these findings. The same group published a follow up study consisting of 310 patients and found that predictors of distant recurrences, peritoneal carcinomatosis and extra pelvic lymph node metastases did not significantly differ and concluded that tumor biology is likely the chief influencer of atypical recurrence, not surgical approach ${ }^{[46]}$. Bochner $\mathrm{et}$ al.$^{\left[{ }^{9]}\right]}$ later found that there was variation in location of recurrence and that RARC resulted in greater numbers of recurrences in the abdomen and pelvis. However, this only achieved significance when pooled and stratification of abdominal recurrences as separate from distant and local recurrences is controversial and of unclear clinical significance ${ }^{[47]}$. Notably, the study was not powered to determine differences in patterns of recurrence. 
Table 3. Recurrence patterns from selected studies

\begin{tabular}{|c|c|c|c|c|c|c|c|c|c|c|}
\hline \multirow[b]{2}{*}{ Ref. } & \multirow[b]{2}{*}{ Year } & \multirow[b]{2}{*}{$\begin{array}{l}\text { Surgical } \\
\text { approach }\end{array}$} & \multirow[b]{2}{*}{$\begin{array}{l}\text { Cases } \\
(n)\end{array}$} & \multirow[b]{2}{*}{$\begin{array}{l}\text { Local } \\
\text { recurrence }{ }^{a} \text {, } \\
\text { n }(\%)\end{array}$} & \multirow[b]{2}{*}{$\begin{array}{l}\text { Distant } \\
\text { recurrence }{ }^{b}, n \\
\text { (\%) }\end{array}$} & \multicolumn{3}{|c|}{ Atypical recurrence $^{c}$} & \multirow[b]{2}{*}{$\begin{array}{l}\text { Significantly } \\
\text { different? }\end{array}$} & \multirow[b]{2}{*}{ Comments } \\
\hline & & & & & & $\begin{array}{l}\text { Peritoneal } \\
\text { carcinomatosis, } n \\
(\%)\end{array}$ & $\begin{array}{l}\text { Abdominal } \\
\text { wall/port site, } \\
n(\%)\end{array}$ & $\begin{array}{l}\text { Extra pelvic } \\
\text { lymph nodes, } \\
n(\%)\end{array}$ & & \\
\hline \multicolumn{11}{|l|}{ Comparative studies, randomized } \\
\hline \multirow{2}{*}{\multicolumn{2}{|c|}{$\begin{aligned} & \text { RAZOR trial, multiple authors } 2020, \\
& 2018,7\end{aligned}$}} & ORC & 152 & $3(2.0)$ & $25(16.4)$ & $1(0.7)$ & $1(0.7)$ & $9(5.9)$ & \multirow[t]{2}{*}{ No } & \multirow[t]{2}{*}{ Largest RCT to date } \\
\hline & & RARC & 150 & $6(4.0)$ & $22(14.7)$ & $2(1.3)$ & 0 & $9(6.0)$ & & \\
\hline \multirow[t]{3}{*}{ CORAL trial, Khan et al. ${ }^{[8]}$} & \multirow[t]{3}{*}{2020} & ORC & 20 & $3(15.0)$ & $\mathrm{nr}$ & $\mathrm{nr}$ & $\mathrm{nr}$ & $\mathrm{nr}$ & \multirow[t]{3}{*}{ No } & \multirow{3}{*}{$\begin{array}{l}\text { Small sample size. Distant } \\
\text { recurrences reported in } \\
\text { aggregate only, not shown here }\end{array}$} \\
\hline & & RARC & 20 & $3(15.0)$ & $\mathrm{nr}$ & $\mathrm{nr}$ & $\mathrm{nr}$ & $\mathrm{nr}$ & & \\
\hline & & LRC & 19 & $3(15.7)$ & $\mathrm{nr}$ & $\mathrm{nr}$ & $\mathrm{nr}$ & $\mathrm{nr}$ & & \\
\hline \multirow[t]{2}{*}{ Bochner et al. ${ }^{[9]}$} & \multirow[t]{2}{*}{2018} & ORC & 58 & $5(8.6)$ & $27(46.6)$ & $2(3.4)$ & 0 & $10(17.2)$ & \multirow[t]{2}{*}{$\mathrm{No}^{\mathrm{d}}$} & \multirow{2}{*}{$\begin{array}{l}\text { Not powered to detect } \\
\text { differences in recurrence } \\
\text { patterns }\end{array}$} \\
\hline & & RARC & 60 & $17(28.3)$ & $20(33.0)$ & $2(3.3)$ & $5(8.3)$ & $5(8.3)$ & & \\
\hline \multicolumn{11}{|c|}{ Comparative studies, non-randomized } \\
\hline \multirow[t]{2}{*}{ Faraj et al. ${ }^{[16]}$} & \multirow[t]{2}{*}{2019} & ORC & 278 & $19(7)$ & $64(23)$ & $5(1.8)$ & 0 & $11(4.0)$ & \multirow[t]{2}{*}{ No } & \multirow[t]{2}{*}{ Large single institutional study } \\
\hline & & RARC & 203 & $12(6)$ & $40(20)$ & $4(2.0)$ & 0 & $4(2.0)$ & & \\
\hline \multirow[t]{2}{*}{ Tan et al. ${ }^{[21]}$} & \multirow[t]{2}{*}{2016} & ORC & 90 & $17(19)$ & $25(28)$ & $3(3)$ & $1(1)$ & $2(2)$ & \multirow[t]{2}{*}{ No } & \multirow{2}{*}{$\begin{array}{l}\text { Intracorporeal diversions in all } \\
\text { robotic cases }\end{array}$} \\
\hline & & RARC & 94 & $11(12)$ & $8(9)$ & $2(2)$ & $1(1)$ & $3(3)$ & & \\
\hline \multirow[t]{2}{*}{ Nguyen et al..$^{[23]}$} & \multirow[t]{2}{*}{2015} & ORC & 79 & $15 / 65(23)$ & $26 / 73(36)$ & $2 / 26(8)$ & $\mathrm{nr}$ & $4 / 26(15)$ & \multirow[t]{2}{*}{ Yes } & Denominator is distant \\
\hline & & RARC & 158 & 24/136 (18) & $43 / 147(29)$ & $9 / 43(21)$ & $\mathrm{nr}$ & $10 / 43(23)$ & & $\begin{array}{l}\text { recurrence, as listed in the } \\
\text { reference }\end{array}$ \\
\hline Non-comparative studies & & & & & & & & & & \\
\hline IRCC, Elsayed et al. ${ }^{[25]}$ & 2021 & RARC only & 2107 & $241(11)$ & $382(18)$ & $26(1.2)$ & $25(1.2)$ & $109(5.2)$ & $\mathrm{n} / \mathrm{a}$ & $\begin{array}{l}\text { RARC not associated with } \\
\text { different patterns or higher } \\
\text { recurrence relative to historic } \\
\text { ORC series }\end{array}$ \\
\hline IRCC, Hussein et al. ${ }^{[27]}$ & 2019 & RARC only & 446 & $69(15)$ & $97(22)$ & $6(1)$ & $5(1)$ & $21(5)$ & $\mathrm{n} / \mathrm{a}$ & $\begin{array}{l}\text { Analysis restricted to patients } \\
\text { with }>10 \text { years follow up }\end{array}$ \\
\hline Collins et al. ${ }^{[29]}$ & 2017 & $\begin{array}{l}\text { RARC, ICUD } \\
\text { only }\end{array}$ & 717 & $78(10.7)$ & $128(17.8)$ & $5(0.7)$ & $2(0.3)$ & $47(6.6)$ & $\mathrm{n} / \mathrm{a}$ & $\begin{array}{l}\text { Totally intracorporeal urinary } \\
\text { diversion cohort }\end{array}$ \\
\hline
\end{tabular}

${ }^{a}$ Local recurrence defined as any recurrence in the cystectomy bed or lymph node dissection template. ${ }^{b}$ Distant recurrence defined as any recurrence which is not local or atypical. ${ }^{c}$ Though sometimes reported in the referenced studies as a subset of distant recurrences, atypical recurrences reported here are mutually exclusive of local and distant recurrence. ${ }^{{ }^{\prime \prime}} T$ The difference in local recurrence rates did not meet conventional levels of significance (sHR $=0.36 ; 95 \% \mathrm{Cl}: 0.11-1.12, P=0.077)$. Similarly, the difference in the rate of abdominal recurrence did not reach statistical significance $(\mathrm{sHR}=0.38 ; 95 \% \mathrm{Cl}: 0.07-1.96 ; P=0.2)$. However, when the pelvic and abdominal recurrences were combined into a single group representing local/regional recurrence, the ORC group showed significantly less local/regional recurrence compared to RARC (sHR = 0.34; 95\%Cl: 0.12-0.93; $P=0.035) "$. RAZOR: randomized open vs. robotic cystectomy; ORC: open radical cystectomy; RARC: robotic assisted radical cystectomy; RCT: randomized controlled trial; CORAL: controlled three-arm trial of open, robotic, and laparoscopic radical cystectomy; IRCC: International Robotic Cystectomy Consortium; ICUD: intracorporeal urinary diversion; nr: not reported. 
Multiple studies have since demonstrated that recurrence patterns do not differ by surgical approach. The RAZOR trial found no significant difference between ORC and RARC in recurrence patterns and showed low overall local recurrence rates ( $2 \%$ vs. $4 \%)$. Rare atypical recurrences were also observed in the ORC arm and did not differ between approaches ${ }^{[7]}$. A large non-randomized single center comparative study from Mayo Clinic in Arizona showed similar rates of local, distant and rare atypical recurrences ${ }^{[16]}$. An institutional report of $\sim 180$ cases, 90 of which were robotic with intracorporeal diversion, showed a low rate of atypical recurrences with no difference between surgical approaches ${ }^{[21]}$. An IRCC study of 2107 pts showed slightly higher local recurrence ( $11 \%$, citing a greater percentage of extravesical disease and variant histology in their cohort) with atypical recurrence patterns similar to ORC series and those of the RAZOR trial ${ }^{[7,25]}$. A separate IRCC analysis found that tumor factors rather than those related to surgical approach were predictive of early recurrence after cystectomy and also showed that surgeons in their cohort reported a very low rate of divergence from oncologic principles ${ }^{[28]}$. Lastly, a large multi-institutional robotic cystectomy and totally intracorporeal urinary diversion cohort from the EAU Robotic Urology Section Scientific Working Group found that early recurrence rates and patterns appeared comparable to open series $^{[22]}$.

If oncologic principles are followed, these aggregate data suggest that atypical recurrence is exceedingly rare and are more likely reflective of tumor biology than surgical approach.

\section{SURVIVAL OUTCOMES}

The primary measure of treatment efficacy in radical cystectomy is survival, including recurrence-free, cancer-specific and overall survival ${ }^{[1]}$. Though reported here for reference, we would discourage direct comparison across studies as there is significant heterogeneity with respect to cancer variables (e.g., receipt of neoadjuvant chemotherapy, disease stage, and tumor histopathology), patient demographic and clinical characteristics, surgeon and institutional factors including intra-operative practices and post-operative follow up protocols, adjuvant therapies and length of follow up. This heterogeneity is reflected by a 2015 systematic review of mostly retrospective studies which demonstrated a wide range of 5-year survival estimates of DFS, CSS and OS between 53\%-74\%, 66\%-80\% and 39\%-66\%, respectively ${ }^{[35]}$.

Several contemporary comparative studies do offer additional limited insight, though we are only aware of 3 RCTs that report survival outcomes. RAZOR is the largest RCT reporting survival outcomes at approximately 150 patients in each arm and reports 3 year outcomes ${ }^{[7]}$. RARC was similar compared with ORC in RFS ( $68 \% v s .65 \%, P=0.6)$ and OS $(74 \% v s .69 \%, P=0.3)$. Bochner et al.${ }^{\left[{ }^{[9]}\right.}$ found that a median follow up of 4.9 years, no differences were observed in recurrence [hazard ratio $(\mathrm{HR})=1.27$; $95 \% \mathrm{CI}$ : 0.69 2.36; $P=0.4$ ], cancer-specific survival $(P=0.4)$, or overall survival $(P=0.8)$. However, the authors cautioned that their study was not powered to assess survival outcomes. A meta-analysis with pooled data from these two studies found that RARC and ORC may result in similar time to recurrence (HR $=1.1$; 95\%CI: 0.8-1.4), but the evidence of certainty was low ${ }^{[37]}$. More recently, the CORAL study reported 5-year RFS, CSS, OS as well and found no differences in surgical approaches comparing open vs. robotic vs. laparoscopic approaches ${ }^{[8]}$. However, their study was limited by low sample size as only 20 patients were included in each arm and included high-risk non-muscle invasive bladder cancer.

Though lacking the rigor of a controlled trial, long-term oncologic outcomes from several robotic cohorts have recently become available. Faraj et al ${ }^{[16]}$ reported their 10 year survival outcomes in a single institution retrospective comparative study and found that RFS and OS were similar between ORC and RARC approaches ( $63 \%$ vs. $70 \%, P=0.14$ and $46 \%$ vs. $40 \%, P=0.47$ respectively). The cohorts were similar in cancer characteristics, patient demographics and clinical factors as well as intra operative practices. 
Retrospective non-comparative results from the IRCC on patients with long-term follow up show RFS, CSS and OS at 10 years were $59 \%, 65 \%$ and $35 \%$, consistent with historical ORC and MIS cohorts ${ }^{[27]}$. Not surprisingly, in multivariable models, they found that survival was associated with age, positive margins, tumor/nodal stage, and adjuvant treatments. Similar results are described in a multicenter study among RARC patients with totally intracorporeal urinary diversion ${ }^{[26]}$. A single institutional comparative study also showed similar survival in a totally intracorporeal urinary diversion robotic cohort when compared with $\mathrm{ORC}^{[18]}$.

Matured, long-term survival data from randomized controlled studies, including RAZOR, are further anticipated. Early and intermediate survival outcomes between RARC and ORC appear to be similar. Since no consistent difference in PSM rates or recurrence patterns have been found in the literature, we expect long-term survival differences to be driven largely by factors related to disease aggressiveness including stage and need for adjuvant therapies, rather than surgical approach.

\section{FUTURE PERSPECTIVES}

Nearly 20 years after the robotic approach to radical cystectomy was described ${ }^{[3]}$. RARC remains an effective and minimally invasive option for patients undergoing cystectomy that can achieve oncologic outcomes that are comparable to the gold standard open approach. Evidence-based consensus and best practices on RARC are available ${ }^{[34]}$.

There are no absolute contraindications to the robotic approach, but an early learning curve is recognized and several challenging case scenarios (e.g., large bulky tumors, history of pelvic radiation) should be preferentially managed by experienced robotic surgeons. RARC can be safely utilized in the octogenarian ${ }^{[48]}$, and oncologic outcomes are excellent in sex-sparing techniques in the female patient ${ }^{[49]}$ as well as male patient ${ }^{[50]}$. Excellent pathologic outcomes have been described for aggressive histopathological variants which are known to present with higher tumor stage ${ }^{[51]}$. The usage of the robotic approach to cystectomy will continue to increase as urologic surgeons become more experienced and comfortable with the platform and education becomes more commonplace in residency training programs $\mathrm{s}^{[4]}$.

Though the current evidence is well-supported, it is limited by the lack of large, randomized controlled trials. We eagerly anticipate more mature, high-quality data comparing oncologic outcomes of open and robotic cystectomy. Robot-assisted radical cystectomy with intracorporeal urinary diversion $v s$. open radical cystectomy (iROC) is a multicenter prospective RCT in England randomizing 320 patients to iRARC or ORC. Accrual finished in February 2020, and oncologic outcomes of interest include atypical recurrence patterns, survival, as well as outcomes related to surgeon fatigue, cost-effectiveness and patient quality of $\operatorname{life}^{[52]}$.

\section{CONCLUSION}

Surgical quality indicators, including lymph node yield and positive surgical margin rate, are comparable between ORC and RARC. Despite an early case series of atypical recurrence patterns, contemporary comparative studies, including the largest randomized controlled trial, as well as a multi-institutional retrospective robotic cohort of $>2000$ consecutive patients, show this is a rare occurrence and not associated with surgical approach. Survival outcomes appear to be similar as well, including long term survival from several comparative and non-comparative reports. Ultimately, surgeon comfort with the selected approach and adherence to oncologic principles is more important than the approach itself. 


\section{DECLARATIONS}

\section{Authors' contributions}

Made substantial contributions to conception and design of the study and performed data acquisition and interpretation: Miller BL, Lau CS, Pachorek M, Yuh B, Sam AP

Performed data acquisition, as well as provided administrative, technical, and material support: Miller BL, Lau CS, Pachorek M

\section{Availability of data and materials}

Not applicable.

\section{Financial support and sponsorship}

None.

\section{Conflicts of interest}

All authors declared that there are no conflicts of interest.

\section{Ethical approval and consent to participate}

Not applicable.

\section{Consent for publication}

Not applicable.

\section{Copyright}

(c) The Author(s) 2021.

\section{REFERENCES}

1. Chang SS, Bochner BH, Chou R, et al. Treatment of non-metastatic muscle-invasive bladder cancer: AUA/ASCO/ASTRO/SUO Guideline. J Urol 2017;198:552-9. DOI PubMed PMC

2. Chang SS, Boorjian SA, et al. Diagnosis and treatment of non-muscle invasive bladder cancer: AUA/SUO Guideline. J Urol 2016;196:1021-9. DOI PubMed

3. Menon M, Hemal AK, Tewari A, et al. Nerve-sparing robot-assisted radical cystoprostatectomy and urinary diversion. BJU Int 2003;92:232-6. DOI PubMed

4. Hu JC, Chughtai B, O'Malley P, et al. Perioperative outcomes, health care costs, and survival after robotic-assisted versus open radical cystectomy: A National Comparative Effectiveness Study. Eur Urol 2016;70:195-202. DOI PubMed

5. Cai PY, Khan AI, Shoag JE, Scherr DS. Robotic radical cystectomy in the contemporary management of bladder cancer. Urol Clin North Am 2021;48:45-50. DOI PubMed

6. Parekh DJ, Reis IM, Castle EP, et al. Robot-assisted radical cystectomy versus open radical cystectomy in patients with bladder cancer (RAZOR): an open-label, randomised, phase 3, non-inferiority trial. Lancet 2018;391:2525-36. DOI PubMed

7. Venkatramani V, Reis IM, Castle EP, et al. Predictors of recurrence, and progression-free and overall survival following open versus robotic radical cystectomy: Analysis from the RAZOR trial with a 3-year followup. J Urol 2020;203:522-9. DOI PubMed PMC

8. Khan MS, Omar K, Ahmed K, et al. Long-term oncological outcomes from an early phase randomised controlled three-arm trial of open, robotic, and laparoscopic radical cystectomy (CORAL). Eur Urol 2020;77:110-8. DOI PubMed

9. Bochner BH, Dalbagni G, Marzouk KH, et al. Randomized trial comparing open radical cystectomy and robot-assisted laparoscopic radical cystectomy: Oncologic outcomes. Eur Urol 2018;74:465-71. DOI PubMed PMC

10. Parekh DJ, Messer J, Fitzgerald J, Ercole B, Svatek R. Perioperative outcomes and oncologic efficacy from a pilot prospective randomized clinical trial of open versus robotic assisted radical cystectomy. J Urol 2013;189:474-9. DOI PubMed

11. Nix J, Smith A, Kurpad R, Nielsen ME, Wallen EM, Pruthi RS. Prospective randomized controlled trial of robotic versus open radical cystectomy for bladder cancer: perioperative and pathologic results. Eur Urol 2010;57:196-201. DOI PubMed

12. Wijburg CJ, Michels CTJ, Hannink G, Grutters JPC, Rovers MM, Alfred Witjes J; RACE Study Group. Robot-assisted radical cystectomy versus open radical cystectomy in bladder cancer patients: a multicentre comparative effectiveness study. Eur Urol 2021;79:609-18. DOI PubMed

13. Asil E, Canda AE, Atmaca AF, et al. Outcomes and complications of radical cystectomy with ileal conduit urinary diversion: A comparison between open, semi-robotic and totally robotic surgery. Int J Med Robot 2021:e2221. DOI PubMed

14. Ip KL, Javier-DesLoges JF, Leung C, et al. Comparison of long-term outcomes in a 10-year experience of robotic cystectomy vs. open cystectomy. J Robot Surg 2020. DOI PubMed 
15. Zhang JH, Ericson KJ, Thomas LJ, et al. Large single institution comparison of perioperative outcomes and complications of open radical cystectomy, intracorporeal robot-assisted radical cystectomy and robotic extracorporeal approach. J Urol 2020;203:512-21. DOI PubMed

16. Faraj KS, Abdul-Muhsin HM, Rose KM, et al. Robot assisted radical cystectomy vs open radical cystectomy: Over 10 years of the Mayo Clinic Experience. Urol Oncol 2019;37:862-9. DOI PubMed

17. Moschini M, Soria F, Mathieu R, et al; European Association of Urology - Young Academic Urologists (EAU-YAU); Urothelial Carcinoma Working Group. Propensity-score-matched comparison of soft tissue surgical margins status between open and roboticassisted radical cystectomy. Urol Oncol 2019;37:179.e171-7. DOI PubMed

18. Simone G, Tuderti G, Misuraca L, et al. Perioperative and mid-term oncologic outcomes of robotic assisted radical cystectomy with totally intracorporeal neobladder: Results of a propensity score matched comparison with open cohort from a single-centre series. Eur J Surg Oncol 2018;44:1432-8. DOI PubMed

19. Hanna N, Leow JJ, Sun M, et al. Comparative effectiveness of robot-assisted vs. open radical cystectomy. Urol Oncol 2018;36:88.e819. DOI PubMed

20. Gandaglia G, Karl A, Novara G, et al. Perioperative and oncologic outcomes of robot-assisted vs. open radical cystectomy in bladder cancer patients: a comparison of two high-volume referral centers. Eur J Surg Oncol 2016;42:1736-43. DOI PubMed

21. Tan WS, Sridhar A, Ellis G, et al. Analysis of open and intracorporeal robotic assisted radical cystectomy shows no significant difference in recurrence patterns and oncological outcomes. Urol Oncol 2016;34:257.e1-9. DOI PubMed

22. Matulewicz RS, DeLancey JO, Manjunath A, Tse J, Kundu SD, Meeks JJ. National comparison of oncologic quality indicators between open and robotic-assisted radical cystectomy. Urol Oncol 2016;34:431.e9-15. DOI PubMed

23. Nguyen DP, Al Hussein Al Awamlh B, Wu X, et al. Recurrence patterns after open and robot-assisted radical cystectomy for bladder cancer. Eur Urol 2015;68:399-405. DOI PubMed PMC

24. Atmaca AF, Canda AE, Gok B, Akbulut Z, Altinova S, Balbay MD. Open versus robotic radical cystectomy with intracorporeal Studer diversion. JSLS 2015;19:e2014.00193. DOI PubMed PMC

25. Elsayed AS, Gibson S, Jing Z, et al. Rates and patterns of recurrences and survival outcomes after robot-assisted radical cystectomy: Results from the International Robotic Cystectomy Consortium. J Urol 2021;205:407-13. DOI PubMed

26. Brassetti A, Cacciamani G, Anceschi U, et al. Long-term oncologic outcomes of robot-assisted radical cystectomy (RARC) with totally intracorporeal urinary diversion (ICUD): a multi-center study. World J Urol 2020;38:837-43. DOI PubMed

27. Hussein AA, Elsayed AS, Aldhaam NA, et al. Ten-year oncologic outcomes following robot-assisted radical cystectomy: Results from the International Robotic Cystectomy Consortium. J Urol 2019;202:927-35. DOI PubMed

28. Hussein AA, Saar M, May PR, et al. Early oncologic failure after robot-assisted radical cystectomy: Results from the International Robotic Cystectomy Consortium. J Urol 2017;197:1427-36. DOI PubMed

29. Collins JW, Hosseini A, Adding C, et al. Early recurrence patterns following totally intracorporeal robot-assisted radical cystectomy: Results from the EAU Robotic Urology Section (ERUS) Scientific Working Group. Eur Urol 2017;71:723-6. DOI PubMed

30. Raza SJ, Wilson T, Peabody JO, et al. Long-term oncologic outcomes following robot-assisted radical cystectomy: results from the International Robotic Cystectomy Consortium. Eur Urol 2015;68:721-8. DOI PubMed

31. Hellenthal NJ, Hussain A, Andrews PE, et al. Lymphadenectomy at the time of robot-assisted radical cystectomy: results from the International Robotic Cystectomy Consortium. BJU Int 2011;107:642-6. DOI PubMed

32. Hellenthal NJ, Hussain A, Andrews PE, et al. Surgical margin status after robot assisted radical cystectomy: results from the International Robotic Cystectomy Consortium. J Urol 2010;184:87-91. DOI PubMed

33. Herr HW, Faulkner JR, Grossman HB, et al. Surgical factors influence bladder cancer outcomes: a cooperative group report. $J$ Clin Oncol 2004;22:2781-9. DOI PubMed

34. Wilson TG, Guru K, Rosen RC, et al. Best practices in robot-assisted radical cystectomy and urinary reconstruction: recommendations of the Pasadena Consensus Panel. Eur Urol 2015;67:363-75. DOI PubMed

35. Yuh B, Wilson T, Bochner B, et al. Systematic review and cumulative analysis of oncologic and functional outcomes after robotassisted radical cystectomy. Eur Urol 2015;67:402-22. DOI PubMed

36. Marshall SJ, Hayn MH, Stegemann AP, et al. Impact of surgeon and volume on extended lymphadenectomy at the time of robotassisted radical cystectomy: results from the International Robotic Cystectomy Consortium (IRCC). BJU Int 2013;111:1075-80. DOI PubMed

37. Rai BP, Bondad J, Vasdev N, et al. Robot-assisted vs open radical cystectomy for bladder cancer in adults. BJU Int 2020;125:765-79. DOI PubMed

38. Sathianathen NJ, Kalapara A, Frydenberg M, et al. Robotic assisted radical cystectomy vs open radical cystectomy: Systematic review and meta-analysis. J Urol 2019;201:715-20. DOI PubMed

39. Dotan ZA, Kavanagh K, Yossepowitch O, et al. Positive surgical margins in soft tissue following radical cystectomy for bladder cancer and cancer specific survival. J Urol 2007;178:2308-12; discussion 2313. DOI PubMed

40. Novara G, Svatek RS, Karakiewicz PI, et al. Soft tissue surgical margin status is a powerful predictor of outcomes after radical cystectomy: a multicenter study of more than 4,400 patients. J Urol 2010;183:2165-70. DOI PubMed

41. Benson MC. Editorial comment. J Urol 2010;184:91. DOI PubMed

42. Karakiewicz PI, Shariat SF, Palapattu GS, et al. Nomogram for predicting disease recurrence after radical cystectomy for transitional cell carcinoma of the bladder. J Urol 2006;176:1354-61; discussion 1361. DOI PubMed

43. Kluth LA, Rieken M, Xylinas E, et al. Gender-specific differences in clinicopathologic outcomes following radical cystectomy: an international multi-institutional study of more than 8000 patients. Eur Urol 2014;66:913-9. DOI PubMed 
44. Sonpavde G, Khan MM, Lerner SP, et al. Disease-free survival at 2 or 3 years correlates with 5-year overall survival of patients undergoing radical cystectomy for muscle invasive bladder cancer. J Urol 2011;185:456-61. DOI PubMed

45. Khetrapal P, Tan WS, Lamb B, et al. Port-site metastases after robotic radical cystectomy: A systematic review and management options. Clin Genitourin Cancer 2017;15:440-4. DOI PubMed

46. Nguyen DP, Al Hussein Al Awamlh B, O'Malley P, et al. Factors impacting the occurrence of local, distant and atypical recurrences after robot-assisted radical cystectomy: A detailed analysis of 310 patients. J Urol 2016;196:1390-6. DOI PubMed

47. Yuh B, Chan K, Wilson T. Robotic cystectomy-moving from innovation to measurable impact. Eur Urol 2018;74:472-3. DOI PubMed

48. Lau CS, Talug J, Williams SB, et al. Robotic-assisted laparoscopic radical cystectomy in the octogenarian. Int J Med Robot 2012;8:247-52. DOI PubMed

49. Tuderti G, Mastroianni R, Flammia S, et al. Sex-sparing robot-assisted radical cystectomy with intracorporeal Padua ileal neobladder in female: Surgical technique, perioperative, oncologic and functional outcomes. J Clin Med 2020;9:577. DOI PubMed PMC

50. Asimakopoulos AD, Campagna A, Gakis G, et al. Nerve sparing, robot-assisted radical cystectomy with intracorporeal bladder substitution in the male. J Urol 2016;196:1549-57. DOI PubMed

51. Koç E, Gök B, Gumuskaya B, Atmaca AF, Canda AE, Balbay MD. Robot assisted radical cystectomy outcomes in micropapillary and plasmacytoid variants. Urol J 2020;17:607-13. DOI PubMed

52. Catto JWF, Khetrapal P, Ambler G, et al. Robot-assisted radical cystectomy with intracorporeal urinary diversion versus open radical cystectomy (iROC): protocol for a randomised controlled trial with internal feasibility study. BMJ Open 2018;8:e020500. DOI PubMed PMC 